\title{
Secondary Hypogonadism at Patients Hormonally Treated for Prostatic Carcinoma from a Cardiologist Perspective
}

\author{
Stefan Farský* \\ Dom srdca Martin, Slovakia
}

*Corresponding author: Stefan Farský, Head cardiologist at Dom srdca Martin, Slovakia.

\begin{abstract}
In the cardiologist's care are often patients treated for erectile dysfunction or prostatic cancer and, vice versa, in the urologist or andrologist's care patients treated for hypertension, arrhythmias and coronary heart disease. There is needed to respect mutually influences and take them in account at the diagnostic and therapeutic indications. Secondary hypogonadism at patients hormonally treated for prostatic carcinoma leads to loss of both positive testosterone effects on cardiovascular and metabolic system and vitality decrease. Cardiovascular rehabilitation carries benefit not to patients with coronary artery disease only, but to patients treated for prostatic carcinoma too. Prostatic disease treatment by urologist may influence blood pressure values of patient treated by cardiologist. This article provides more detail information's on the pathological-physiological background of relevant mutual relationships and their practical impact on clinical patient management.
\end{abstract}

Keywords: Prostatic carcinoma, Hypogonadism and cardiovascular disease, Cardiovascular rehabilitation

\section{Introduction}

Hormonal treatment of $\mathrm{Ca}$ prostate leads to secondary hypogonadism, a marked drop in testosterone levels that occurs in human serum in three forms: free, easily bound to albumin or strongly bound to sex hormone-binding globulin (SHBG). Testosterone receptors are found to be ubiquitous in the human body, and the effects of testosterone are crucial to male life [1].

a. Improving quality and life expectancy

b. Increases sexual desire and improves erectile function

c. Increase muscle mass, muscle strength and bone density, increase blood formation and physical activity

d. Reduce visceral fat volume and risk of fractures

e. Improves the feeling of mental well-being, mood, memory, vitality

Of particular importance are the positive effects of testosterone and its modulators (LHRH hormone releasing luteinizing hormone) on the cardiovascular system [2-4]. a. Modulate myocardial contractility (receptors in myocytes)

b. Vasodilator effects on coronary artery disease, inhibition of endothelial cell apoptosis

c. Antiarrhythmic effects, shortening of the QTc interval

d. Inhibition of atherosclerosis, improvement of insulin resistance, improvement of metabolic syndrome dyslipidemia, decrease of glucose level and TK

e. Immunomodulatory effects (decrease in cytokine levels associated with inflammation and development of atherosclerosis)

f. Low testosterone levels are associated with increased cardiovascular mortality

Most patients treated for prostate cancer are of higher age, with a high prevalence of cardiovascular disease or middle age when high cardiovascular (CV) risk is possible. Hormonal therapy leads to secondary hypogonadism, which may additionally be 
potentiated by natural aging, metabolic syndrome or some other drugs (antiarrhythmics, spironolactones, steroids, etc.). And for cardiologists a cardinal question arises: to indicate hormonal treatment that will favor the underlying malignancy, but on the other hand will cause a significant decrease in testosterone levels and the loss of all beneficial effects associated with patients with high KV risk or already developed KV disease?

It is necessary to stratify patients from the point of view of $\mathrm{KV}$ and metabolic risk, way of life, possibility of injuries, etc. In a urological or andrological ambulance under time pressure, the doctor sometimes just asks "did you overcome the heart attack?", Which is totally inadequate. Andrology is used to assess the state of the state from the point of view of the burden of sexual contact, The Princeton III Consensus Conference Questionnaire [5]. Low-risk patients are asymptomatic in normal activity, including in sexual intercourse, with 2 shoulders rising within 10 seconds or walking $1.5 \mathrm{~km}$ within 20 minutes. Moderate risk factors include patients with mild stable angina pectoris, peripheral atherosclerosis of lower limb atherosclerosis, post-stroke or transient ischemic attack, heart failure in the NYHA class 3 . These patients need to perform echocardiographic and physical pharmacological burden (Table 1). According to the results, the patient is reclassified and can be included in controlled cardio training [6]. High risk is present in patients with complex arrhythmias (stress-induced ventricular tachycardia or multiple ventricular extrasystoles, uncontrolled atrial fibrillation), uncontrolled hypertension, unstable angina pectoris, aortic valve stenosis, hypertrophic cardiomyopathy, heart failure in the NYHA 4 functional class. needed cardiological counseling.

Table 1: Cardiovascular risk assessment based on exercise test and echocardiographic examination results.

\begin{tabular}{|c|c|}
\hline Low risk & $\begin{array}{c}\text { EF LV } \geq 45 \% \text {, without ischemic ECG changes before and } \\
\text { during exercise, without arrhytmias, exercise capacity }> \\
100 \mathrm{~W}(>6 \mathrm{MET})\end{array}$ \\
\hline Middle risk & $\begin{array}{c}\text { EF LV 31-44 \%, ischemic ECG changes at higher intensity } \\
\text { of exercise }(>100 \mathrm{~W}), \text { exercise capacity }<100 \mathrm{~W}(<6 \\
\text { METs) }\end{array}$ \\
\hline High risk & $\begin{array}{l}\text { EF LV }<30 \%, \text { ventricular arrhytmias, systolic BP decrease } \\
\text { during exercise more than } 10 \text { mm Hg, significant ischemic } \\
\text { changes at low intensity of exercise or in basal conditions. }\end{array}$ \\
\hline
\end{tabular}

EF LV - left ventricle ejection fraction, METs - metabolic equivalents

Stratification of patients by KV risk in sexual intercourse is helpful in deciding whether hormonal therapy is used in patients with Ca prostate and should be part of standard diagnostic and therapeutic procedures. Nevertheless, it does not even reveal a high risk for asymptomatic patients, as evidenced by many cases of sudden "full-health" deaths. Therefore, in the so- of suspect patients, usually in the presence of strong risk factors (smoking, obesity, stress), should not be lagging with the indication of coronary imaging methods (CT coronary angiography, invasive coronary angiography) or with indication of long-term cardiac rhythm monitoring.

In addition, the personality of the patient and his or her way of life is an important moment when deciding. Many adverse HP and metabolic effects of hypogonadism may be impaired by proper diet, diet, and especially cardio training.
Potential of controlled cardiothorization in mortality of Ca prostate patients and on the reduction of adverse effects of hormonal therapy

The potential for regular and effective physical training can be nicely illustrated in the morbidity of middle-aged patients (on average 38 years) with low testosterone levels surviving on platinum-based treatment for testicular carcinoma [7]. These patients can expect long-term survival after diagnosis and treatment of oncological disease, but also with all the negative effects of hypogonadism. $3 \mathrm{x}$ more often are treatments for dyslipidemia, 2 more often for diabetes mellitus, hypertension and erectile dysfunction, $1.5 \mathrm{x}$ more often for anxiety and depression. Overall, they have a 7-fold higher risk of disease than those of the normal population. Although the risk of developing hypogonadism was influenced by genetic factors, it is noteworthy that intensive regular physical activity demonstrated a protective effect prior to hypogonadism $(\mathrm{p}=0.06)$.

Both American and European cardiology companies and the American Cancer Society recommend adults 75 minutes of intensive or 150minutes of moderate physical weekly activities. Wang et al. performed an analysis of men with non-metastatic Ca prostate who were observed for nearly 20 years under the "Prostate Cancer Prevention Trial, PCPT". Approximately 7,300 patients who regularly practiced Ca-prostate diagnostics and 5,300 patients who regularly practiced after diagnosis were included in the analysis. Exercise intensity was evaluated in metabolic equivalent hours (METs hours) that were different for running, jogging, walking, cycling, swimming, dancing. They found that those who practiced 17.5 METs hours or more a week before diagnosing oncological disease had a significantly lower risk of Ca prostate death than those who trained less than 8.75 hours METs per week $[8,9]$. Low risk was defined as stage T1-T2 and Gleason score 2-7. The protective effect of exercise before diagnosis has not been demonstrated for patients with a high-risk type of Ca prostate. But if these high-risk patients intensively trained (more than 17.5 METS hours per week) after the diagnosis, the risk of CA prostate death was also significantly reduced.

Adverse effects of hypogonadism during or after hormonal therapy of CA prostate can also be reduced by controlled cardio training. These patients have reduced muscle and bone mass, performance, cognitive and sexual function, increased body fat content, increased fatigue, and impaired laboratory metabolic parameters in terms of metabolic syndrome. With a controlled, long-lasting exercise combined with aerobic and resistance training, they can achieve an overall improvement in quality of life, cardiorespiratory performance, muscle mass and muscle strength compared to similar patients who have only received general press releases on the need for changing life and regular exercise.

\section{Acknowledgement}

None.

\section{Conflict of Interest}

No Conflict of interest. 


\section{References}

1. Marenčák J (2017) The clinical significance of testosterone in an aging man from a urologist perspective. Via practica 14(2): 80-86.

2. Poljak Z, Fillo J, Aziriová S (2015) Cardiovascular complications of hormonal treatment of prostate cancer. Monitor medicíny SLS 1(2): 8-12.

3. Charbit B, Christin Maitre S, Démolis JL, Soustre E, Young J, et al. (2009) Effects of testosterone on ventricular repolarization in hypogonadic men. Am J Cardiol 103(6): 887-890.

4. Webb CM, McNeill JG, Hayward CS, de Zeigler D, Collins P (1999) Effects of testosterone on coronary vasomotor regulation in men with coronary heart disease. Circulation 100(16): 1690-1696.

5. Nehra A, Jackson G, Miner M, Billups KL, Burnett AL, et al. (2012) The Princeton III Consensus recommendations for the management of erectile dysfunction and cardiovascular disease. Mayo Clin Proc 87(8): $766-768$.
6. Farský Š, Rus V, Sládeková K (2017) How to perform an outpatient cardiovascular rehabilitation: the proposal of the Working Group of Cardiovascular Rehabilitation of Slovak cardiological society. Inside Lék 63 (12): 969-976.

7. Abu Zaid MI, Gathirua Mwangi WG, Williams AL, et al. (2017) Metabolic syndrome after platinum-based chemotherapy: A multicenter study of North American testicular cancer survivors. 2017 Cancer Survivorship Symposium of the American Society of Clinical Oncology.

8. Wang Y, Jacobs EJ, Gapstur SM, Maliniak ML, Gansler T, et al. (2017) Recreational physical activity in relation to prostate cancer-specific mortality among men with nonmetastatic prostate cancer. Eur Urol 72 (6): 931-939.

9. Galvao DA, Spry N, Denham J, Taaffe DR, Cormie P, et al. (2014) A multicentre year-long randomized controlled trial of physical exercise in men with prostate cancer previously treated with androgen suppression and radiation from TROG 03.04 RADAR. Eur Urol 65(5): 856-864. 